\title{
On the Probabilistic Degree of OR over the Reals
}

\section{Siddharth Bhandari}

TIFR, Mumbai, India

siddharth.bhandari@tifr.res.in

\section{Prahladh Harsha}

TIFR, Mumbai, India

prahladh@tifr.res.in

\section{Tulasimohan Molli}

TIFR, Mumbai, India

tulasi.molli@tifr.res.in

\section{Srikanth Srinivasan}

Department of Mathematics, IIT Bombay, Mumbai, India srikanth@math.iitb.ac.in

\section{Abstract}

We study the probabilistic degree over $\mathbb{R}$ of the OR function on $n$ variables. For $\varepsilon \in(0,1 / 3)$, the $\varepsilon$-error probabilistic degree of any Boolean function $f:\{0,1\}^{n} \rightarrow\{0,1\}$ over $\mathbb{R}$ is the smallest non-negative integer $d$ such that the following holds: there exists a distribution of polynomials $\mathbf{P} \in \mathbb{R}\left[x_{1}, \ldots, x_{n}\right]$ entirely supported on polynomials of degree at most $d$ such that for all $z \in\{0,1\}^{n}$, we have $\operatorname{Pr}_{P \sim \mathbf{P}}[P(z)=f(z)] \geq 1-\varepsilon$. It is known from the works of Tarui (Theoret. Comput. Sci. 1993) and Beigel, Reingold, and Spielman (Proc. 6th CCC 1991), that the $\varepsilon$-error probabilistic degree of the OR function is at most $O(\log n \cdot \log (1 / \varepsilon))$. Our first observation is that this can be improved to $O(\log (\underset{\leq \log (1 / \varepsilon)}{n}))$, which is better for small values of $\varepsilon$.

In all known constructions of probabilistic polynomials for the OR function (including the above improvement), the polynomials $P$ in the support of the distribution $\mathbf{P}$ have the following special structure:

$$
P\left(x_{1}, \ldots, x_{n}\right)=1-\prod_{i \in[t]}\left(1-L_{i}\left(x_{1}, \ldots, x_{n}\right)\right),
$$

where each $L_{i}\left(x_{1}, \ldots, x_{n}\right)$ is a linear form in the variables $x_{1}, \ldots, x_{n}$, i.e., the polynomial $1-P(\bar{x})$ is a product of affine forms. We show that the $\varepsilon$-error probabilistic degree of OR when restricted to polynomials of the above form is $\Omega\left(\log \left(\begin{array}{c}n \\ \leq \log (1 / \varepsilon)\end{array}\right) / \log ^{2}\left(\log \left(\begin{array}{c}n \\ \leq \log (1 / \varepsilon))\end{array}\right)\right)\right)$, thus matching the above upper bound (up to polylogarithmic factors).

2012 ACM Subject Classification Theory of computation $\rightarrow$ Probabilistic computation, Theory of computation $\rightarrow$ Circuit complexity

Keywords and phrases Polynomials over reals, probabilistic polynomials, probabilistic degree, OR polynomial

Digital Object Identifier 10.4230/LIPIcs.FSTTCS.2018.5

Acknowledgements The authors thanks Noga Alon for referring them to the paper on radiobroadcast [1].

(c) (i) Siddharth Bhandari, Prahladh Harsha, Tulasimohan Molli, and Srikanth Srinivasan;

cc. licensed under Creative Commons License CC-BY

38th IARCS Annual Conference on Foundations of Software Technology and Theoretical Computer Science (FSTTCS 2018).

Editors: Sumit Ganguly and Paritosh Pandya; Article No. 5; pp. 5:1-5:12 


\section{Introduction}

Low-degree polynomial approximations of Boolean functions were introduced by Razborov in his celebrated work [11] on proving lower bounds for the class of Boolean functions computed by low-depth circuits. We begin by recalling this notion of approximation over $\mathbb{R}$.

- Definition 1.1 (probabilistic degree). Given a Boolean function $f:\{0,1\}^{n} \rightarrow\{0,1\}$ and $\varepsilon \in(0,1 / 3)$, an $\varepsilon$-error probabilistic polynomial over $\mathbb{R}^{1}$ for $f$ is a distribution of polynomials $\mathbf{P}\left(x_{1}, \ldots, x_{n}\right) \in \mathbb{R}\left[x_{1}, \ldots, x_{n}\right]$ such that for any $z \in\{0,1\}^{n}$, we have $\operatorname{Pr}_{P \sim \mathbf{P}}[P(z) \neq f(z)] \leq \varepsilon$. The $\varepsilon$-error Probabilistic degree of $f$, denoted by $\mathrm{P}-\operatorname{deg}_{\varepsilon}(f)$, is the smallest non-negative integer $d$ such that the following holds: there exists an $\varepsilon$-error probabilistic polynomial $\mathbf{P}$ over $\mathbb{R}$ such that $\mathbf{P}$ is entirely supported on polynomials of degree at most $d$.

Classical results in polynomial approximation of Boolean functions $[15,14,3]$ show that the OR function over $n$ variables, denoted by $\mathrm{OR}_{n}$, has $\varepsilon$-error probabilistic degree at most $O(\log n \cdot \log (1 / \varepsilon))$. This basic construction for the OR function is then recursively used to show that any function computed by an $\mathrm{AC}^{0}$ circuit of size $s$ and depth $d$ has $\varepsilon$-error probabilistic degree at most $(\log s)^{O(d)} \cdot \log (1 / \varepsilon)$ (see work by the second and last author [6] for recent improvements). These results can then be used to prove, eg. [12], a (slightly weaker) version of Håstad's celebrated theorem [7] that parity does not have subexponential-sized $\mathrm{AC}^{0}$ circuits. These results were employed more recently by Braverman [4] to prove that polylog-wise independence fools $\mathrm{AC}^{0}$ functions.

Despite the fact that probabilistic polynomials for the OR function are such a basic primitive, it is surprising that we do not yet have a complete understanding of $\mathrm{P}-\mathrm{deg}_{\varepsilon}\left(\mathrm{OR}_{n}\right)$. As mentioned above, it is known from the works of Beigel, Reingold and Spielman [3] and Tarui [14] that $\mathrm{P}-\operatorname{deg}_{\varepsilon}\left(\mathrm{OR}_{n}\right)=O(\log n \cdot \log (1 / \varepsilon))$. It can be easily checked via a simple application of the Schwartz-Zippel lemma that a dependence of $\Omega(\log (1 / \varepsilon))$ is necessary in the above bound. However, till not long ago, it was unclear if any dependence on $n$ is required over the reals ${ }^{2}$. In recent papers of Meka, Nguyen and $\mathrm{Vu}$ [10] and the second and last author [6], it was shown using anti-concentration of low-degree polynomials that the $\mathrm{P}-\operatorname{deg}_{1 / 4}\left(\mathrm{OR}_{n}\right)=\widetilde{\Omega}(\sqrt{\log n})$. The main objective of this paper is to obtain a better understanding of the $\varepsilon$-error probabilistic degree of $\mathrm{OR}_{n}, \mathrm{P}-\mathrm{deg}_{\varepsilon}(\mathrm{OR} n)$. Besides being interesting in its own right, this question has bearing on the amount of independence needed to fool $\mathrm{AC}^{0}$ circuits. Recent improvements due to Tal [13] and [6] of Braverman's result demonstrate that $(\log s)^{2.5 d+O(1)} \cdot \log (1 / \varepsilon)$-wise independence fools functions computed by $\mathrm{AC}^{0}$ circuits of size $s$ and depth $d$. An improvement of the upper bound on $\mathrm{P}-\mathrm{deg}_{\varepsilon}\left(\mathrm{OR}_{n}\right)$ to $O(\log n)+\log (1 / \varepsilon))$ could potentially strengthen this result to $(\log s)^{d+O(1)} \cdot \log (1 / \varepsilon)$, nearly matching the lower bound of $(\log s)^{d-1} \cdot \log (1 / \varepsilon)$ due to Mansour [9].

The above discussion demonstrates that the current bounds on $\mathrm{P}-\operatorname{deg}_{\varepsilon}\left(\mathrm{OR}_{n}\right)$ fall short of being tight in two aspects: one, the dependence on $n$ in the lower bound is $\widetilde{\Omega}(\sqrt{\log n})$ while in the upper bound it is $O(\log n)$ and two, the joint dependence on $\varepsilon$ and $n$ in the upper bound is multiplicative, i.e., $O(\log n \cdot \log (1 / \varepsilon))$ while the current lower bounds can only show an additive $\widetilde{\Omega}(\sqrt{\log n})+\Omega(\log (1 / \varepsilon))$ bound.

Which of these bounds is tight? A casual observer might suspect that the upper bound is, given the relatively neat expression. However, a closer look tells us that it cannot be, at least when $\varepsilon$ is quite small. For example, setting $\varepsilon=1 / 2^{\Omega(n)}$, the upper bound yields a

1 Similar notions over other fields are also studied. Unless otherwise specified, we will be considering probabilistic polynomials over the reals in this paper.

2 For finite fields of constant size, Razborov [11] showed that the $\varepsilon$-error probabilistic degree of $\mathrm{OR}_{n}$ is $O(\log (1 / \varepsilon))$, independent of $n$, the number of the input bits. 
degree of $O(n \log n)$, but it is a standard fact that any Boolean function on $n$ variables can be represented exactly (i.e. with no error) as a polynomial of degree $n$. Hence the upper bound is not tight in this regime.

Our first observation is that the upper bound of Tarui and Beigel et al. [3] can indeed be slightly improved to $O\left(\log \left(\begin{array}{c}n \\ \leq \log (1 / \varepsilon))\end{array}\right)\right) ;^{3}$ note that this is asymptotically better than $O(\log n \cdot \log (1 / \varepsilon))$ for very small $\varepsilon$. This interpolates smoothly between the construction of Tarui [14] and Beigel et al. [3] and the exact representation of degree $n$ mentioned above. (See Section 2 for details on this upper-bound construction.)

Given this observation, one might hope to prove a matching lower bound on the $\varepsilon$-error probabilistic degree of $\mathrm{OR}_{n}$. We can indeed show such a bound (upto polylogarithmic factors) if we suitably restrict the class of polynomials being considered. While restricted, this subclass of polynomials nevertheless includes all polynomials that were used in previous upper bound constructions, including our own. Moreover, this result generalizes a result of Alon, Bar-Noy, Linial and Peleg [1], who prove such a result for a further restricted class of polynomials (mentioned at the end of this section) and for $\log (1 / \varepsilon)=O(\log n) .{ }^{4}$ A careful reworking of their analysis shows that their lower bound extends to even smaller $\varepsilon$ to show a lower bound of $\Omega\left(\log \left(\begin{array}{c}n \\ \leq \log (1 / \varepsilon)\end{array}\right)\right)$ for this smaller class of polynomials.

To state our result, we first need to describe the class of polynomials for which our bounds hold. To this end, we note that all known upper-bound constructions of probabilistic polynomials for the OR function have the following structure:

$$
P\left(x_{1}, \ldots, x_{n}\right)=1-\prod_{i \in[t]}\left(1-L_{i}\left(x_{1}, \ldots, x_{n}\right)\right),
$$

where each $L_{i}\left(x_{1}, \ldots, x_{n}\right)=a_{i 1} x_{1}+a_{i 2} x_{2}+\cdots+a_{i n} x_{n}$ is a linear form in the variables $x_{1}, \ldots, x_{n}$ (here, $\left.a_{i j} \in \mathbb{R}\right)$.

This includes the improved upper-bound construction that achieves an $\varepsilon$-probabilistic degree of $O\left(\log \left(\begin{array}{c}n \\ \leq \log (1 / \varepsilon)\end{array}\right)\right)$ mentioned in the preceding paragraph. This motivates the following definition.

- Definition 1.2 (hyperplane covering polynomials). A polynomial $P \in \mathbb{R}\left[x_{1}, \ldots, x_{n}\right]$ is said to be a hyperplane covering polynomial of degree $t$ if there exist $t$ linear forms $L_{1}, \ldots, L_{t}$ over the reals such that

$$
P\left(x_{1}, \ldots, x_{n}\right)=1-\prod_{i \in[t]}\left(1-L_{i}\left(x_{1}, \ldots, x_{n}\right)\right) .
$$

For $\varepsilon \in(0,1 / 2)$, the $\varepsilon$-error hyperplane covering probabilistic degree of $f$, denoted by $\mathrm{hcP}-\operatorname{deg}_{\varepsilon}(f)$, is the smallest non-negative integer $d$ such that the following holds: there exists an $\varepsilon$-error probabilistic polynomial $\mathbf{P}$ over $\mathbb{R}$ such that $\mathbf{P}$ is supported on hyperplane covering polynomials of degree at most $d$.

We call these polynomials hyperplane covering polynomials as these polynomials have the property that the one's of the polynomials in the Boolean hypercube (i.e, the set $\{z \in$ $\left.\left.\{0,1\}^{n} \mid P(z)=1\right\}\right)$ are a union of hyperplanes not passing through the origin. We further note that all these polynomials satisfy the property that $P(\overline{0})=0$. Clearly, hcP- $\operatorname{deg}_{\varepsilon}(f) \geq$

\footnotetext{
3 Here, $\left(\begin{array}{c}N \\ \leq \alpha\end{array}\right)$ denotes $\sum_{i<\alpha}\left(\begin{array}{c}N \\ i\end{array}\right)$.

4 The result of [1] is stated in a slightly different language, but is essentially equivalent to a probabilistic degree lower bound for $\mathrm{OR}_{n}$ for a suitable class of polynomials.
} 
P- $\operatorname{deg}_{\varepsilon}(f)$. Also, since all upper-bound constructions for the OR polynomials are hyperplane covering polynomials, we not only have that $\mathrm{P}-\operatorname{deg}_{\varepsilon}\left(\mathrm{OR}_{n}\right)=O(\log (\underset{\leq \log (1 / \varepsilon)}{n}))$ but also that hcP- $-\operatorname{deg}_{\varepsilon}\left(\mathrm{OR}_{n}\right)=O(\log (\underset{\leq \log (1 / \varepsilon)}{n}))$. For this class of polynomials, we prove the following (almost) tight result on the $\varepsilon$-error hyperplane covering probabilistic degree of the OR function.

- Theorem 1.3 (hyperplane covering degree of $\mathrm{OR}_{n}$ ). For any any positive integer $n$ and $\varepsilon \in(0,1 / 3)$,

$$
\mathrm{hcP}-\operatorname{deg}_{\varepsilon}\left(\mathrm{OR}_{n}\right)=\Omega\left(\frac{\log \left(\begin{array}{c}
n \\
\leq \log (1 / \varepsilon)
\end{array}\right)}{\log ^{2}\left(\log \left(\begin{array}{c}
n \\
\leq \log (1 / \varepsilon)
\end{array}\right)\right)}\right) .
$$

It is open if this result can be extended to prove a tighter lower bound on the $\varepsilon$-error probabilistic degree of the $\mathrm{OR}_{n}$ function. The special class of hyperplane covering polynomials for which Alon, Bar-Noy, Peleg and Linial [1] proved a similar bound is the class of hyperplane covering polynomials where the linear forms are sums of variables (i.e., $L_{i}(\bar{z})=\sum_{j \in S_{i}} z_{j}$ for some $S_{i} \subseteq[n]$ ). Ideally, one would have liked to extend their lower bound result for hyperplace covering polynomials where the linear forms are sums of variables to all polynomials. Theorem 1.3, is a step in this direction, in that, it shows that their result can be extended to a slightly larger class, the set of all hyperplane covering polynomials (modulo polylogarithmic factors). We remark that though our lower bound works for a larger class of polynomials, our proof technique is nevertheless inspired by their proof.

\section{Upper bounds on probabilistic degree of OR}

In this section, we describe the construction of a probabilistic polynomial which shows that the hcP- $\operatorname{deg}_{\varepsilon}\left(\mathrm{OR}_{n}\right)=O\left(\log \left(\begin{array}{c}n \\ \leq \log (1 / \varepsilon)\end{array}\right)\right)$. To begin with, we observe that the following "trivial" hyperplane covering polynomial of degree $n$ exactly computes $\mathrm{OR}_{n}$ everywhere on the Boolean hypercube:

$$
P_{\mathrm{OR}}(x):=1-\prod_{i=1}^{n}\left(1-\frac{1}{i} \sum_{j \in[n]} x_{j}\right) .
$$

This is a polynomial which covers each Hamming slice of the hypercube with a different hyperplane. We now recall the construction of Beigel, Reingold and Spielman [3] and Tarui [14].

- Claim 2.1. For every non-negative integer $\ell$, there exists a distribution of linear forms $\mathbf{L}_{\ell}$ such that if the Hamming weight of $x=\left(x_{1}, \ldots, x_{n}\right)$ lies in the interval $\left[2^{\ell}, 2^{\ell+1}\right]$, then $\operatorname{Pr}_{L \sim \mathbf{L}}[L(x)=1]=\Omega(1)$.

Proof. $\mathbf{L}$ is defined as follows: pick a random set $S \subseteq[n]$ by picking each element of $[n]$ independently with probability $\frac{1}{2^{\ell}}$ and construct the linear polynomial

$$
L_{S}(x):=\sum_{i \in S} x_{i}
$$


For a non-zero input $x=\left(x_{1}, \ldots, x_{n}\right)$ such that the Hamming weight of $x$, denoted as $|x|$, is in $\left[2^{\ell}, 2^{\ell+1}\right]$, we have

$$
\begin{array}{rlrl}
\operatorname{Pr}_{S}\left[L_{S}(x)=1\right] & =|x|\left(\frac{1}{2^{\ell}}\right)\left(1-\frac{1}{2^{\ell}}\right)^{|x|-1} & & {\left[\text { where } 0^{0}=1\right]} \\
& =\frac{|x|}{2^{\ell}} \exp (-O(1)) & & {\left[\because(1-a)^{b} \geq \exp (-a b / 1-a)\right]} \\
& \geq \Omega(1) . &
\end{array}
$$

In the above proof we could have set $L_{S}(x)=\sum_{i \in S} \alpha_{i} x_{i}$ where each $\alpha_{i} \in \pm 1$ u.a.r. and independently. Clearly, even with the new definition $\operatorname{Pr}_{S}\left[L_{S}(x)=1\right] \geq \Omega(1)$. The idea behind introducing the $\alpha$ 's is that even when $\sum_{i \in S} x_{i}>1$, it could be that $\sum_{i \in S} \alpha_{i} x_{i}=1$. However, this does not lead to improvements beyond possibly changing the constant hidden in the $\Omega(\cdot)$ notation.

The preceding claim is then used to construct $\varepsilon$-error probabilistic polynomials for $\mathrm{OR}_{n}$ as follows. Divide the set of one's of the OR function in the Boolean hypercube, ie., $\{0,1\}^{n} \backslash\{\overline{0}\}$, into $\log n$ epochs $\left[2^{0}, 2^{1}\right],\left[2^{1}, 2^{2}\right], \ldots,\left[2^{\log n-1}, 2^{\log n}\right]$ where each epoch $\left[2^{\ell}, 2^{\ell+1}\right]$ includes all strings whose Hamming weight is in that range. For each such epoch $\left[2^{\ell}, 2^{\ell+1}\right]$, sample $t:=O(\log (1 / \varepsilon))$ independent linear forms $L_{i}^{(\ell)}, i \in[t]$ from $\mathbf{L}_{\ell}$ and consider the randomized polynomial $P_{\ell}(x):=1-\prod_{i \in[t]}\left(1-L_{i}^{(\ell)}(x)\right)$. Clearly, for $x$ in the epoch $\left[2^{\ell}, 2^{\ell+1}\right]$, we have $\operatorname{Pr}\left[P_{\ell}(x)=1\right] \geq 1-\varepsilon$. Now, the randomized polynomial

$$
P(x):=1-\prod_{\ell \in[\log n]}\left(1-P_{\ell}(x)\right)=1-\prod_{\ell \in[\log n]} \prod_{i \in[t]}\left(1-L_{i}^{(\ell)}\right),
$$

satisfies for all $x \in\{0,1\}^{n} \backslash\{\overline{0}\}, \operatorname{Pr}[P(x)=1] \geq 1-\varepsilon$. Also, clearly any such $P$ satisfies $P(\overline{0})=$ 0 . This polynomial is a hyperplane covering polynomial of degree at most $O(\log n \cdot \log (1 / \varepsilon))$.

Now, suppose $\varepsilon$ is very small, eg., $\varepsilon=2^{-n / 10}$, then this construction is wasteful over the trivial construction $P_{\mathrm{OR}}$ since $O(\log n \cdot \log (1 / \varepsilon))=O(n \log n)$. The improved bound of $O\left(\log \left(\begin{array}{c}n \\ \leq \log (1 / \varepsilon))\end{array}\right)\right.$ is obtained by "interpolating" between the trivial construction $P_{\mathrm{OR}}$ and the above construction. Since we know that the $\mathrm{P}-\operatorname{deg}_{\varepsilon}\left(\mathrm{OR}_{n}\right)$ is at least $\log (1 / \varepsilon)$, one might as well exactly compute $\mathrm{OR}_{n}$ for the first $O(\log (1 / \varepsilon))$ Hamming slices of the hypercube and use the above randomized construction to cover the remaining slices using only $(\log n-\log \log (1 / \varepsilon))$ epochs, $[\log (1 / \varepsilon), 2 \log (1 / \varepsilon)], \ldots,\left[2^{\log n-1}, 2^{\log n}\right]$. Another way to view this is that when we focus on the epoch $\left[2^{\ell}, 2^{\ell+1}\right]$ and draw $t=O(\log (1 / \varepsilon))$ samples from $\mathbf{L}_{\ell}$, the trivial polynomial $\prod_{i=2^{\ell}}^{2^{\ell+1}}\left(1-\frac{1}{i} \sum_{j \in[n]} x_{j}\right)$ has degree smaller than $O(\log (1 / \varepsilon))$ when $2^{\ell}<\log (1 / \varepsilon)$ or $\ell<\log \log (1 / \varepsilon)$.

Formally, we construct the polynomial (where $P_{\ell}(x)$ and $L^{(\ell)}$ are as defined above)

$$
\begin{aligned}
P(x) & :=1-\left(\prod_{\ell \in[\log \log (1 / \varepsilon), \log n]}\left(1-P_{\ell}(x)\right)\right) \times \prod_{i=1}^{\log (1 / \varepsilon)}\left(1-\frac{1}{i} \sum_{j \in[n]} x_{j}\right) \\
& =1-\left(\prod_{\ell \in[\log \log (1 / \varepsilon), \log n]} \prod_{i \in[t]}\left(1-L_{i}^{(\ell)}\right)\right) \times \prod_{i=1}^{\log (1 / \varepsilon)}\left(1-\frac{1}{i} \sum_{j \in[n]} x_{j}\right) .
\end{aligned}
$$

Clearly, $P$ is a hyperplane covering polynomial. For an input $x$ such that $|x| \leq \log (1 / \varepsilon)$, $P(x)=1$ as $\prod_{i=1}^{\log (1 / \varepsilon)}\left(1-\frac{1}{i} \sum_{j \in[n]} x_{j}\right)=0$. If $|x| \in\left[2^{\ell}, 2^{\ell+1}\right]$ where $\ell \geq \log \log (1 / \varepsilon)$, then from our previous argument we have $\operatorname{Pr}\left[P_{l}(x)=1\right] \geq 1-\varepsilon$ and hence $\operatorname{Pr}[P(x)=1] \geq 1-\varepsilon$. Hence, we have an $\varepsilon$-error probabilistic polynomial of degree $O(\log (1 / \varepsilon)+(\log n-\log \log (1 / \varepsilon))$. $\log (1 / \varepsilon))$ which is at most $O\left(\log \left(\begin{array}{c}n \\ \leq \log (1 / \varepsilon)\end{array}\right)\right)$. 


\section{Lower bound on hyperplane covering degree of OR}

We now turn to the lower bound. To prove a lower bound of $d_{\varepsilon}:=\widetilde{\Omega}\left(\log \left(\begin{array}{c}n \\ \leq \log (1 / \varepsilon)\end{array}\right)\right)$, by Yao's minmax theorem (duality arguments) it suffices (and is neccesary) to demonstrate a "hard" distribution $\mathcal{D}_{\varepsilon}$ under which it is hard to approximate the $\mathrm{OR}_{n}$ function by any hyperplane covering polynomial of degree at most $d_{\varepsilon}$.

Similar to previous works $[10,6]$, our choice of hard distribution is motivated by the polynomial constructions in the upper bound. We first need the following definitions to define the hard distribution $\mathcal{D}_{\varepsilon}$.

- Definition $3.1\left((0,1)\right.$-restriction $\left.\mu_{p}\right)$. The $\mu_{p}^{[n]}$ distribution on $\{0,1\}^{n}$ is obtained by setting each variable $x_{i}$ independently to 1 with probability $p$ and 0 otherwise.

- Definition $3.2\left((0, *)\right.$-restriction $\left.\rho_{p}\right)$. The $\rho_{p}^{[n]}$ distribution on $\{0, *\}^{n}$ is obtained by setting each variable to 0 independently with probability $(1-p)$ and leaving it unset with probability $p$.

If the number of variables is $n$, we will drop the superscript and refer to the corresponding restrictions as just $\mu_{p}$ and $\rho_{p}$ respectively.

It will be convenient to view the distribution $\mu_{p}$ as applying a $(0, *)$ restriction $\rho_{2 p}$ followed by a $\{0,1\}$ restriction $\mu_{1 / 2}$ to the unset variables. In short, $\mu_{p}^{[n]}=\mu_{1 / 2}^{\rho_{2 p}^{-1}(*)} \circ \rho_{2 p}^{[n]}$.

- Definition 3.3 (hard distribution). Consider the distribution $\mathcal{D}_{\varepsilon}$ on the input set $\{0,1\}^{n}$ defined as follows:

- pick an integer $\ell \in I_{\varepsilon}:=[1, \log n-\log \log (1 / \varepsilon)] \cap \mathbb{Z}$ uniformly at random.

- pick $x \in\{0,1\}^{n}$ according to $\mu_{1 / 2} \ell$, i.e., for each $i \in[n]$, independently sets $x_{i} \leftarrow 1$ with probability $1 / 2^{\ell}$ and 0 otherwise.

The hard distribution $\mathcal{D}_{\varepsilon}$ is a convex combination of the distributions $\mu_{1 / 2^{\ell}}$ for $\ell \in I_{\varepsilon}$. In other words, $\mathcal{D}_{\varepsilon}:=\frac{1}{\left|I_{\varepsilon}\right|} \sum_{\ell \in I_{\varepsilon}} \mu_{1 / 2^{\ell}}$. Each of the distributions $\mu_{1 / 2^{\ell}}$ roughly correspond to the epochs used in the upper-bound construction.

Theorem 1.3 follows from the following "distributional" version of the theorem.

- Theorem 3.4. Let $\mathcal{D}_{\varepsilon}$ be the hard distribution defined in Definition 3.3 and $P=1$ $\prod_{i \in[t]}\left(1-L_{i}\right)$ be a hyperplane covering polynomial of degree $t$ such that

$\operatorname{Pr}_{x \sim \mathcal{D}_{\varepsilon}}\left[P(x) \neq O R_{n}(x)\right] \leq \varepsilon$

then, $t \geq \Omega\left(\frac{\log \left(\begin{array}{c}n \\ \leq \log (1 / \varepsilon)\end{array}\right)}{\log ^{2}\left(\log \left(\begin{array}{l}n \\ \leq \log (1 / \varepsilon)\end{array}\right)\right)}\right)$.

We now introduce some notations that will be useful. For a set $S,|S|$ denotes the cardinality of $S$, and for an input $x \in 0,1^{n},|x|$ denotes the Hamming weight of $x$.

- Definition 3.5 (support of a linear form). For a linear form $L\left(x_{1}, \ldots, x_{n}\right)=a_{1} x_{1}+a_{2} x_{2}+$ $\cdots+a_{n} x_{n}$, we define the support of $L$, denoted as $\operatorname{supp}(L)$, to be the set of non-zero $a_{i}$ 's, i.e., $\left|\left\{i \in[n] \mid a_{i} \neq 0\right\}\right|$.

The proof of Theorem 3.4 requires the following variant of the Schwartz-Zippel Lemma (due to Alon and Füredi [2]) and Littlewood-Offord-Erdös' anti-concentration lemma of linear forms over the reals, which we state below. 
- Lemma 3.6 ([2, Theorem 5]). Let $P \in \mathbb{R}\left[x_{1}, \ldots, x_{n}\right]$ be a polynomial of degree at most $d$ polynomial over $\mathbb{R}$ computing a non-zero function over $\{0,1\}^{n}$. Then for $x$ chosen uniformly from $\{0,1\}^{n}$,

$$
\operatorname{Pr}_{x \in\{0,1\}^{n}}[P(x) \neq 0] \geq \frac{1}{2^{d}} .
$$

- Lemma 3.7 (anti-concentration of linear forms over $\mathbb{R}[8,5])$. Let $L\left(x_{1}, \ldots, x_{k}\right)=\sum a_{i} x_{i}$ be a linear form which is supported on exactly $k$ variables (i.e., $a_{i} \neq 0, i=1, \ldots, k$. Then, for all $a \in \mathbb{R}$ and $x$ chosen uniformly from $\{0,1\}^{n}$,

$$
\operatorname{Pr}_{x \in\{0,1\}^{n}}\left[L_{i}(x)=a\right] \leq \frac{1}{\sqrt{k}} .
$$

The rest of this section is devoted to proving Theorem 3.4. We begin with a proof outline in Section 3.1 followed by the proof in Section 3.2.

\subsection{Proof outline}

We would like to show that hyperplane covering polynomial $P$ that approximates $\mathrm{OR}_{n}$ w.r.t distribution $\mathcal{D}_{\varepsilon}$ (as in Theorem 3.4) must have large degree. Let $\mathcal{L}$ denote the set of linear forms that appear in $P$, i.e., $\mathcal{L}:=\left\{L_{i} \mid i \in[t]\right\}$.

Let us see how $P$ behaves on the distribution $\mu_{1 / 2^{\ell}}$ or equivalently $\mu_{1 / 2} \circ \rho_{1 / 2^{\ell-1}}$. Let us see what happens to the linear forms $\left\{L_{i}, i \in[t]\right\}$ when the restriction $\rho:=\rho_{1 / 2^{\ell-1}}$ is first applied. We first consider two extreme cases.

Very few linear forms survive: Suppose all but $\log (1 / 2 \varepsilon)$ linear forms trivialize on the restriction $\rho$ (i.e. the corresponding linear form $\left.L_{i}\right|_{\rho}$ becomes 0$)$. Then, $\left.(1-P)\right|_{\rho}$ is a polynomial of degree at most $\log (1 / 2 \varepsilon)$ computing a non-zero function (since $1-P(\overline{0})=1$ ). Hence, by Lemma 3.6, it is not equal to 0 with probability at least $2 \varepsilon$. This implies that the polynomial $P$ errs with probability at least $2 \varepsilon$ on the distribution $\mu_{1 / 2^{\ell}}$.

All linear forms that survive have large support: Suppose all the linear forms that survive post restriction $\rho$ have large support, say $4 t^{2}$. Then, by the anti-concentration of linear forms over reals (Lemma 3.7), we have that each linear form is 1 with probability at most $1 / \sqrt{4 t^{2}}=1 / 2 t$. Since there are most $t$ linear forms, the probability that any of them is 1 is at most $t / 2 t=1 / 2$. Thus, $P$ errs with probability $1 / 2$ on the distribution $\mu_{1 / 2^{\ell}}$.

Note that the actual situation for each distribution $\mu_{1 / 2^{\ell}}$ will most likely be a combination of the above two. We can then show that a combination of the above two arguments will still work if the surviving linear forms have the following nice structure. Let $\mathcal{L}_{\rho}$ be the set of surviving linear forms subsequent to the restiction $\rho$, i.e., $\mathcal{L}_{\rho}:=\left\{\left.L_{i}\right|_{\rho}\left|i \in[t], L_{i}\right|_{\rho} \neq 0\right\}$. Suppose $\mathcal{L}_{\rho}$ can be partitioned into 2 sets $\mathcal{L}_{\rho}^{\prime} \dot{\cup} \mathcal{L}_{\rho}^{\prime \prime}$ such that the number of linear forms in $\mathcal{L}_{\rho}^{\prime}$ is small (less than $O(\log (1 / \varepsilon))$ ) and each of the linear forms in $\mathcal{L}_{\rho}^{\prime \prime}$ have large support even after subtracting $\cup_{L \in \mathcal{L}_{\rho}^{\prime}} \operatorname{supp}(L)$ from their support. How does one then show that a constant faction of $\rho$ 's satisfy that the corresponding linear forms $\mathcal{L}_{\rho}$ have this nice structure? For this, we draw inspiration from the proof of Alon, Bar-Noy, Linial and Peleg [1], where they prove similar bounds for hyperplane covering polynomials supported entirely on linear forms arising as sums of variables. They construct an appropriate potential function that guarantees a similar property in their lower-bound argument.

We use a slightly different potential function, which has the following nice property. If the total number of linear forms is $t$, then $\mathbb{E}_{\ell}\left[\Phi_{\ell}(\mathcal{L})\right]=O(t /(\log n-\log \log (1 / \varepsilon)))$ and furthermore, whenever $\Phi_{\ell}(\mathcal{L})$ is small then the corresponding set $\mathcal{L}_{\ell}$ of surviving linear forms post restriction $\rho_{1 / 2^{\ell-1}}$ can be partitioned as indicated above. This shows that for most $\ell, P$ errs on computing the $\mathrm{OR}_{n}$ function unless $t$ is large. 


\subsection{Proof of Theorem 3.4}

We now turn to defining the potential function $\Phi_{\ell}(\mathcal{L})$, indicated in the proof outline.

- Definition 3.8 (potential function). The weight of a linear form $L$, denoted by $w(L)$, is defined as follows:

$$
w(L):= \begin{cases}0 & \text { if } \operatorname{supp}(L)=\emptyset, \\ \frac{1}{\log ^{2}(2|\operatorname{supp}(L)|)} & \text { otherwise. }\end{cases}
$$

Given a collection $\mathcal{L}=\left\{L_{1}, \ldots, L_{t}\right\}$ of linear forms and $\ell$ a positive integer, the potential function $\Phi_{\ell}(\mathcal{L})$ is defined as follows

$$
\Phi_{\ell}(\mathcal{L}):=\sum_{i=1}^{t} \underset{\rho_{1 / 2}^{\ell-1}}{\mathbb{E}}\left[w\left(\left.L_{i}\right|_{\rho_{1 / 2}^{\ell-1}}\right)\right]
$$

where $\rho_{1 / 2^{\ell-1}}$ is a $(0, *)$-restriction as defined in Definition 3.2.

The potential function $\Phi_{\ell}(\mathcal{L})$ satisfies the following two properties, given by Propositions 3.9 and 3.10 .

- Proposition 3.9. There exists a universal constant $C$ such that the following holds. Let $\mathcal{L}=\left\{L_{1}, \ldots, L_{t}\right\}$ be any collection of $t$ linear forms, then

$$
\underset{\ell \in I_{\varepsilon}}{\mathbb{E}}\left[\Phi_{\ell}(\mathcal{L})\right] \leq \frac{C t}{\left|I_{\varepsilon}\right|}
$$

- Proposition 3.10 (partition of linear forms). Let $\mathcal{L}=\left\{L_{1}, \ldots, L_{t}\right\}$ be a collection of $t$ non-zero linear forms and $K, R$ be two positive integers such that

$$
\sum_{i=1}^{t} w\left(L_{i}\right)<\frac{R}{\log ^{2}(2 R K)} .
$$

Then, there exists a partition $\mathcal{L}=\mathcal{L}^{\prime} \dot{\cup} \mathcal{L}^{\prime \prime}$ of the set of linear forms $\mathcal{L}$ such that

- $\left|\mathcal{L}^{\prime}\right| \leq R$,

- For all $L \in \mathcal{L}^{\prime \prime},\left|\operatorname{supp}(L) \backslash \cup_{L^{\prime} \in \mathcal{L}^{\prime}} \operatorname{supp}\left(L^{\prime}\right)\right| \geq K$.

Before proving these two propositions, we first show how they imply Theorem 3.4.

Proof of Theorem 3.4. Let

$$
t:=\frac{\log (1 / 8 \varepsilon) \cdot(\log n-\log \log (1 / \varepsilon))}{2 C \log ^{2}\left(1 / C^{2} \cdot \log ^{4}(1 / 8 \varepsilon) \cdot(\log n-\log \log (1 / \varepsilon))^{3}\right)},
$$

where $C$ is the universal constant in Proposition 3.9. Clearly, $t=\Omega\left(\frac{\log \left(\begin{array}{c}n \\ \leq \log (1 / \varepsilon)\end{array}\right)}{n}\right)$. Let $P=1-\prod_{i \in[t]}\left(1-L_{i}\right)$ be any hyperplane covering polynomial of degree $t$. To prove the theorem, it suffices if we show that $\operatorname{Pr}_{x \sim \mathcal{D}_{\varepsilon}}\left[P(x) \neq \mathrm{OR}_{n}(x)\right]>\varepsilon$. To this end, we first note that $\operatorname{Pr}_{x \sim \mathcal{D}_{\varepsilon}}[x=\overline{0}]<\varepsilon$ (since for all $\ell \in I_{\varepsilon}$, we have $\ell \leq \log n-\log \log (1 / \varepsilon)$ ). Hence, to prove the theorem it suffices to show that $\operatorname{Pr}_{x \sim \mathcal{D}_{\varepsilon}}[P(x) \neq 1] \geq 2 \varepsilon$.

Since $\mathcal{D}_{\varepsilon}=\frac{1}{\left|I_{\varepsilon}\right|} \sum_{\ell \in I_{\varepsilon}} \mu_{1 / 2^{\ell}}$ and $\mu_{p}^{[n]}=\mu_{1 / 2} \circ \rho_{2 p}^{[n]}$, this is equivalent to showing

$$
\underset{\ell \in I_{\varepsilon}}{\mathbb{E}}\left[\underset{\rho \sim \rho_{1 / 2}^{\ell-1}}{\mathbb{E}}\left[\underset{x \sim \mu_{1 / 2}}{\operatorname{Pr}}\left[\left.P\right|_{\rho}(x) \neq 1\right]\right]\right] \geq 2 \varepsilon
$$


To this end, we first apply Proposition 3.9 to the set $\mathcal{L}$ of $t$ linear forms in the polynomial $P$ to obtain that

$$
\underset{\ell \in I_{\varepsilon}}{\mathbb{E}}\left[\underset{\rho \sim \rho_{1 / 2^{\ell-1}}}{\mathbb{E}}\left[\sum_{i \in[t]} w\left(\left.L_{i}\right|_{\rho}\right)\right]\right]=\underset{\ell \in I_{\varepsilon}}{\mathbb{E}}\left[\Phi_{\ell}(\mathcal{L})\right] \leq \frac{C t}{\left|I_{\varepsilon}\right|} .
$$

Applying Markov to the above inequality, we have

$$
\operatorname{Pr}_{\ell, \rho}\left[\sum_{i \in[t]} w\left(\left.L_{i}\right|_{\rho}\right) \leq \frac{2 C t}{\left|I_{\varepsilon}\right|}\right] \geq \frac{1}{2} .
$$

We call an $(\ell, \rho)$ pair good if the above event holds, i.e., $\sum_{i=1}^{t} w\left(\left.L_{i}\right|_{\rho}\right) \leq 2 C t /\left|I_{\varepsilon}\right|$. Thus,

$$
\underset{\ell, \rho}{\operatorname{Pr}}[(\ell, \rho) \text { is } \operatorname{good}] \geq 1 / 2 .
$$

Now given a good $(\ell, \rho)$-pair, let $\mathcal{L}_{\rho}$ be the set of surviving linear forms subsequent to the restiction $\rho$, i.e., $\mathcal{L}_{\rho}:=\left\{\left.L_{i}\right|_{\rho}\left|i \in[t], L_{i}\right|_{\rho} \neq 0\right\}$. We thus have $\sum_{L \in \mathcal{L}_{\rho}} w(L) \leq 2 C t /\left|I_{\varepsilon}\right|$. Let $K:=4 t^{2}$ and $R:=\log (1 / 8 \varepsilon)$. It can be checked that for this choice of parameters we have $2 C t /\left|I_{\varepsilon}\right|<R / \log ^{2}(2 R K)$. We can now apply Proposition 3.10 to obtain a partition $\mathcal{L}_{\rho}=\mathcal{L}_{\rho}^{\prime} \dot{\cup} \mathcal{L}_{\rho}^{\prime \prime}$ such that

- $\left|\mathcal{L}_{\rho}^{\prime}\right| \leq R=\log (1 / 8 \varepsilon)$,

- for all $L \in \mathcal{L}_{\rho}^{\prime \prime}$, we have $\left|\operatorname{supp}(L) \backslash \cup_{L^{\prime} \in \mathcal{L}_{\rho}^{\prime}} \operatorname{supp}\left(L^{\prime}\right)\right| \geq K=4 t^{2}$.

Consider the polynomial $\left.P\right|_{\rho}=1-\prod_{i \in[t]}\left(1-\left.L_{i}\right|_{\rho}\right)=1-\prod_{\left.L \in \mathcal{L}\right|_{\rho}}(1-L)$ subsequent to the restriction $\rho$. We will rewrite this polynomial as $\left.P\right|_{\rho}=1-Q_{\rho}^{\prime} \cdot Q_{\rho}^{\prime \prime}$ where the polynomials $Q_{\rho}^{\prime}$ and $Q_{\rho}^{\prime \prime}$ are defined as follows (using the sets $\mathcal{L}_{\rho}^{\prime}$ and $\mathcal{L}_{\rho}^{\prime \prime}$ respectively).

$$
\begin{aligned}
& Q_{\rho}^{\prime}(x):=\prod_{L \in \mathcal{L}_{\rho}^{\prime}}(1-L(x)), \\
& Q_{\rho}^{\prime \prime}(x):=\prod_{L \in \mathcal{L}_{\rho}^{\prime \prime}}(1-L(x)) .
\end{aligned}
$$

Clearly, $\left.P\right|_{\rho}=1-Q_{\rho}^{\prime} \cdot Q_{\rho}^{\prime \prime}$.

Since $\left|\mathcal{L}_{\rho}^{\prime}\right| \leq \log (1 / 8 \varepsilon)$, we have that the degree of $Q_{\rho}^{\prime}$ is at most $\log (1 / 8 \varepsilon)$. Furthermore $Q_{\rho}^{\prime}(x) \not \equiv 0$ (since $Q_{\rho}^{\prime}(\overline{0})=1$ ). Thus applying Lemma 3.6, we have

$$
\operatorname{Pr}_{x \sim \mu_{1 / 2}}\left[Q_{\rho}^{\prime}(x) \neq 0\right] \geq 8 \varepsilon .
$$

Consider any setting of variables in $\cup_{L \in \mathcal{L}_{\rho}^{\prime}} \operatorname{supp}(L)$ such that $Q_{\rho}^{\prime}(x) \neq 0$. Even conditioned on setting all these variables, we know that each $L \in \mathcal{L}_{\rho}^{\prime \prime}$ still has surviving support of size at least $4 t^{2}$. Thus, by Lemma 3.7, we have for each $L \in \mathcal{L}_{\rho}^{\prime \prime}$,

$$
\operatorname{Pr}_{x \sim \mu_{1 / 2}}\left[L(x)=1 \mid Q_{\rho}^{\prime}(x) \neq 0\right] \leq \frac{1}{\sqrt{4 t^{2}}}=\frac{1}{2 t} .
$$

By a union bound, we have

$$
\operatorname{Pr}_{x \sim \mu_{1 / 2}}\left[Q_{\rho}^{\prime \prime}(x)=0 \mid Q_{\rho}^{\prime}(x) \neq 0\right]=\operatorname{Pr}_{x \sim \mu_{1 / 2}}\left[\exists L \in \mathcal{L}_{\rho}^{\prime \prime}, L(x)=1 \mid Q_{\rho}^{\prime}(x) \neq 0\right] \leq \frac{t}{2 t}=\frac{1}{2} .
$$

Hence,

$$
\operatorname{Pr}_{x \sim \mu_{1 / 2}}\left[\left.P\right|_{\rho}(x) \neq 1\right]=\operatorname{Pr}\left[Q_{\rho}^{\prime}(x) \neq 0\right] \cdot \operatorname{Pr}\left[Q_{\rho}^{\prime \prime}(x)=0 \mid Q_{\rho}^{\prime}(x) \neq 0\right] \geq 8 \varepsilon \cdot \frac{1}{2}=4 \varepsilon .
$$


Finally averaging over all $(\ell, \rho)$ we have from above and $(2)$

$$
\operatorname{Pr}_{x \sim \mathcal{D}_{\varepsilon}}[P(x) \neq 1] \geq \operatorname{Pr}_{\ell, \rho}[(\ell, \rho) \text { is good }] \cdot \operatorname{Pr}\left[\left.P\right|_{\rho}(x) \neq 1 \mid(\ell, \rho) \text { is good }\right] \geq \frac{1}{2} \cdot 4 \varepsilon=2 \varepsilon .
$$

This proves (1) and thus completes the proof of Theorem 3.4.

We are now left with the proofs of Propositions 3.9 and 3.10. We begin with the proof of Proposition 3.10.

Proof of Proposition 3.10. Consider the following algorithm to obtain the partition $\mathcal{L}=$ $\mathcal{L}^{\prime} \cup \mathcal{L}^{\prime \prime}$

1. Initialize $\mathcal{L}^{\prime} \leftarrow \emptyset$ and $\mathcal{L}^{\prime \prime} \leftarrow \mathcal{L}$.

2. While there exists an $L \in \mathcal{L}^{\prime \prime}$ such that $\left|\operatorname{supp}(L) \backslash \cup_{L^{\prime} \in \mathcal{L}^{\prime}} \operatorname{supp}\left(L^{\prime}\right)\right| \leq K$,

- Move such an $L$ from $\mathcal{L}^{\prime \prime}$ to $\mathcal{L}^{\prime}$ (i.e., $\mathcal{L}^{\prime} \leftarrow \mathcal{L}^{\prime} \cup\{L\}$ and $\mathcal{L}^{\prime \prime} \leftarrow \mathcal{L}^{\prime \prime} \backslash\{L\}$ ).

Clearly, when the algorithm terminates, we have $\left|\operatorname{supp}(L) \backslash \operatorname{supp}\left(\mathcal{L}^{\prime}\right)\right| \geq K$ for all $L \in \mathcal{L}^{\prime \prime}$.

We now argue that $\left|\mathcal{L}^{\prime}\right| \leq R$. Each iteration of the while loop adds a linear form $L$ to $\mathcal{L}^{\prime}$ with at most $K$ new variables. If the while loop is performed for $T$ iterations, then the support of each $L$ added to $\mathcal{L}^{\prime}$ is at most $T K$. We now argue that $T<R$. If not, then after exactly $R$ iterations of the while loop, we have that

$$
\sum_{L \in \mathcal{L}} w(L) \geq \sum_{L \in \mathcal{L}^{\prime}} w(L) \geq \frac{R}{\log ^{2}(2 R K)}
$$

contradicting the hypothesis of the proposition. Hence $T<R$. The size of $\mathcal{L}^{\prime}$ is the number of iterations of the while loop and is thus bounded above by $R$. This completes the proof of the proposition.

\section{Proof of Proposition 3.9.}

$$
\begin{aligned}
\underset{\ell \in I_{\varepsilon}}{\mathbb{E}}\left[\Phi_{\ell}(\mathcal{L})\right] & =\underset{\ell \in I_{\varepsilon}}{\mathbb{E}}\left[\underset{\rho \sim \rho_{1 / 2} \ell_{-1}}{\mathbb{E}}\left[\sum_{i \in[t]} w\left(\left.L_{i}\right|_{\rho}\right)\right]\right] \\
& =\frac{1}{\left|I_{\varepsilon}\right|} \sum_{i \in[t]} \sum_{\ell \in I_{\varepsilon}} \underset{\rho}{\mathbb{E}}\left[w\left(\left.L_{i}\right|_{\rho}\right)\right] \\
& \leq \frac{1}{\left|I_{\varepsilon}\right|} \sum_{i \in[t]}(\underbrace{\sum_{\ell>\log \left|\operatorname{supp}\left(L_{i}\right)\right|} \underset{\rho}{\mathbb{E}}\left[w\left(\left.L_{i}\right|_{\rho}\right)\right]}_{T_{1}}+\underbrace{\sum_{\ell \leq \log \left|\operatorname{supp}\left(L_{i}\right)\right|}^{\mathbb{E}[}\left[w\left(\left.L_{i}\right|_{\rho}\right)\right]}_{T_{2}}) .
\end{aligned}
$$

$T_{1}$ and $T_{2}$ are bound using Claim 3.11 and Claim 3.12 respectively. Hence,

$$
\underset{\ell \in I_{\varepsilon}}{\mathbb{E}}\left[\Phi_{\ell}(\mathcal{L})\right] \leq \frac{1}{\left|I_{\varepsilon}\right|} \sum_{i=1}^{t}\left(2+\frac{\pi^{2}}{6}+\frac{e}{e-1}\right) \leq \frac{t}{\left|I_{\varepsilon}\right|} \cdot\left(3+\frac{\pi^{2}}{6}\right) .
$$

- Claim 3.11. Let $L$ be a linear form such that $|\operatorname{supp}(L)|=k$. Then

$$
\sum_{\ell: \ell>\log k} \underset{\rho \sim \rho_{1 / 2}^{\ell-1}}{\mathbb{E}}\left[w\left(\left.L\right|_{\rho}\right)\right] \leq 2
$$


Proof.

$$
\begin{aligned}
& \sum_{\ell: \ell>\log k} \underset{\rho \sim \rho_{1 / 2}^{\ell-1}}{\mathbb{E}}\left[w\left(\left.L\right|_{\rho}\right)\right] \\
& \leq \sum_{\ell: \ell>\log k}\left(\underset{\rho}{\operatorname{Pr}}\left[\left|\operatorname{supp}\left(\left.L\right|_{\rho}\right)\right|=0\right] \cdot 0+\underset{\rho}{\operatorname{Pr}}\left[\left|\operatorname{supp}\left(\left.L\right|_{\rho}\right)\right| \geq 1\right] \cdot 1\right) \\
& \leq \sum_{\ell: \ell>\log k}\left(1-\left(1-\frac{1}{2^{\ell-1}}\right)^{k}\right) \\
& \leq \sum_{\ell: \ell>\log k} \frac{k}{2^{\ell-1}} \\
& {\left[\because(1-x)^{n} \geq 1-n x, \forall 0<x \leq 1\right]} \\
& \leq 2 \text {. }
\end{aligned}
$$

Claim 3.12. Let $L$ be a linear form such that $|\operatorname{supp}(L)|=k$. Then

$$
\sum_{\ell \leq \log k} \underset{\rho \sim \rho_{1 / 2}^{\ell-1}}{\mathbb{E}}\left[w\left(\left.L\right|_{\rho}\right)\right] \leq \frac{\pi^{2}}{6}+\frac{e}{e-1}
$$

Proof.

$$
\begin{aligned}
& \underset{\rho}{\mathbb{E}}\left[w\left(\left.L\right|_{\rho}\right)\right] \leq \underset{\rho}{\operatorname{Pr}}\left[\left|\operatorname{supp}\left(\left.L\right|_{\rho}\right)\right| \geq \frac{k}{2^{\ell}}\right] \frac{1}{\log ^{2}\left(k / 2^{\ell}\right)}+\underset{\rho}{\operatorname{Pr}}\left[\left|\operatorname{supp}\left(\left.L\right|_{\rho}\right)\right| \leq \frac{1}{2} \cdot \frac{k}{2^{\ell-1}}\right] \\
& \leq \quad \text { [by chernoff bound] } \\
& \sum_{\ell \leq \log k} \underset{\rho \sim \mathcal{R}_{\ell}}{\mathbb{E}}\left[w\left(\left.L\right|_{\rho}\right)\right] \leq \sum_{\ell \leq \log (k)} \frac{1}{(\log (k)-\ell)^{2}}+\sum_{\ell \leq \log (k)} \exp \left(-\frac{k}{2^{\ell+1}}\right) \\
& \leq \frac{\pi^{2}}{6}+\frac{e}{e-1} .
\end{aligned}
$$

\section{References}

1 Noga Alon, Amotz Bar-Noy, Nathan Linial, and David Peleg. A Lower Bound for Radio Broadcast. J. Comput. Syst. Sci., 43(2):290-298, 1991. doi:10.1016/0022-0000(91) 90015-W.

2 Noga Alon and Zoltán Füredi. Covering the Cube by Affine Hyperplanes. Eur. J. Comb., 14(2):79-83, 1993. doi:10.1006/eujc.1993.1011.

3 Richard Beigel, Nick Reingold, and Daniel A. Spielman. The Perceptron Strikes Back. In Proc. 6th IEEE Conf. on Structure in Complexity Theory, pages 286-291, 1991. doi: 10.1109/SCT.1991.160270.

4 Mark Braverman. Polylogarithmic independence fools $A C^{0}$ circuits. J. ACM, 57(5), 2010. (Preliminary version in 24th IEEE Conference on Computational Complexity, 2009). eccc: 2009/TR09-011, doi:10.1145/1754399.1754401.

5 Paul Erdős. On a lemma of Littlewood and Offord. Bull. Amer. Math. Soc., 51(12):898-902, 1945. doi:10.1090/S0002-9904-1945-08454-7.

6 Prahladh Harsha and Srikanth Srinivasan. On Polynomial Approximations to $A C^{0}$. Random Structures Algorithms, 2018. (Preliminary version in 20th RANDOM, 2016). doi:10.1002/rsa. 20786. 
7 Johan Håstad. Almost optimal lower bounds for small depth circuits. In Silvio Micali, editor, Randomness and Computation, volume 5 of Advances in Computing Research, pages 143170. JAI Press, Greenwich, Connecticut, 1989. (Preliminary version in 18th STOC 1986). URL: http://www.csc.kth.se/ johanh/largesmalldepth.pdf.

8 John Edensor Littlewood and A. Cyril Offord. On the Number of Real Roots of a Random Algebraic Equation. J. London Math. Soc., s1-13(4):288-295, 1938. doi:10.1112/jlms/ s1-13.4.288.

9 Michael Luby and Boban Velickovic. On Deterministic Approximation of DNF. Algorithmica, 16(4/5):415-433, 1996. (Preliminary version in 23rd STOC, 1991). doi: 10.1007/BF01940873.

10 Raghu Meka, Oanh Nguyen, and Van Vu. Anti-concentration for Polynomials of Independent Random Variables. Theory Comput., 12(11):1-17, 2016. doi : 10.4086/toc. 2016. v012a011.

11 Alexander A. Razborov. Нжние оценки размера схем ограниченной глубины в полном базисе, содержащем функцию логического сложения (Russian) [Lower bounds on the size of bounded depth circuits over a complete basis with logical addition]. Mathematicheskie Zametki, 41(4):598-607, 1987. (English translation in Mathematical Notes of the Academy of Sciences of the USSR, 41(4):333-338, 1987). doi:10.1007/BF01137685.

12 Roman Smolensky. Algebraic Methods in the Theory of Lower Bounds for Boolean Circuit Complexity. In Proc. 19th ACM Symp. on Theory of Computing (STOC), pages 77-82, 1987. doi:10.1145/28395.28404.

13 Avishay Tal. Tight Bounds on the Fourier Spectrum of $A C^{0}$. In Proc. 32nd Comput. Complexity Conf., volume 79 of LIPIcs, pages 15:1-15:31. Schloss Dagstuhl, 2017. eccc: 2014/TR14-174, doi:10.4230/LIPIcs.CCC.2017.15.

14 Jun Tarui. Probabilistic Polynomials, $A C^{0}$ Functions, and the Polynomial-Time Hierarchy. Theoret. Comput. Sci., 113(1):167-183, 1993. (Preliminary version in 8th STACS, 1991). doi : 10.1016/0304-3975(93) 90214-E.

15 Seinosuke Toda and Mitsunori Ogiwara. Counting Classes are at Least as Hard as the Polynomial-Time Hierarchy. SIAM J. Comput., 21(2):316-328, 1992. (Preliminary version in 6th Structure in Complexity Theory Conference, 1991). doi:10.1137/0221023. 